\title{
TWO SIDED SAND PILES MODEL AND UNIMODAL SEQUENCES
}

\author{
THI HA DUONG PHAN ${ }^{1}$
}

\begin{abstract}
We introduce natural generalizations of two well-known dynamical systems, the Sand Piles Model and the Brylawski's model. We describe their order structure, their reachable configuration's characterization, their fixed points and their maximal and minimal length's chains. Finally, we present an induced model generating the set of unimodal sequences which amongst other corollaries, implies that this set is equipped with a lattice structure.
\end{abstract}

Mathematics Subject Classification. 68R05, 05A17.

\section{INTRODUCTION}

Sand Piles Model (SPM) and several related models such as Chip Firing Game or Brylawski's model have been introduced and studied in various contexts. They were used to illustrate the self-organized criticality paradigm in physics by Bak et al. [2], and appear in the study of legal game sequences [4], in language theory by Bjorner and Ziegler [5], and of course, in combinatorics (see, for example, Anderson et al. [1], Spencer [18] and Goles and Kiwi [11]).

In our study, a discrete dynamical model is defined by the space of configurations and certain transition rule(s). The goal is to investigate whether the model converges to a stable configuration (called fixed point), and if it converges, then what the number of transitions needed is. It is also important to obtain a criterion for which a configuration is reachable from another by repeated applications of the given transition rules. To describe the set of all reachable configurations we use

Keywords and phrases. Discrete dynamical system, Sand Piles Model, partition, unimodal sequence, order, lattice, dominance ordering, fixed point.

1 LIAFA Université Denis Diderot, Paris 7 - Case 7014-2, Place Jussieu- 75256 Paris Cedex 05-France and Institute of Mathematics, 18 Hoang Quoc Viet, Hanoi, Vietnam;

phan@liafa.jussieu.fr; phanhaduong@math.ac.vn 
combinatorial objects such as partitions, unimodal sequences, and to illustrate the transitions, we use some special structures such as order and lattice.

In the context of Sand Piles Model, a configuration is a sequence of sand piles such that their heights are decreasing from left to right. For a given total number of sand grains $n$, each configuration can be represented by a partition of $n$ [11]. The transition rule is the following: at each moment, one sand grain can fall down from one pile to its right neighbor with the condition that the resulted configuration is still a decreasing sequence. This model defines a partial order over the set of integer partitions of $n$. On the other hand, Brylawski's model $\left(L_{B}\right)$ was introduced in 1973 [7] to represent the dominance ordering over the set of all integer partitions of a given integer. This model can be viewed as a generalization of $S P M$ by adding one transition rule: at each moment, one sand grain can slide from one pile to another pile in its right (not necessary its neighbor). The two posets obtained by $S P M$ and by $L_{B}$ have lattice structure with many interesting properties [11-15].

Inspired by the meaningful results of these models, many generalizations of $S P M$ were introduced and studied. For example, a parallel $S P M$ was studied in [3], next model $I P M$ was defined by relaxing transition rule [13], and recently a bidimentional $S P M$ was introduced [9].

In this paper, we generalize these two classical models in a natural way by considering transitions in both sides, that means one grain can fall down in either directions, from right to left or also from left to right. The generalized models are called "General Sand Piles Model" $(G S P M)$ and "General $L_{B}$ " $\left(G L_{B}\right)$ respectively. It turns out that these two models are closely related to the notion of unimodality, a subject also occurs in many branches of mathematics and computer science. For a survey of unimodal sequences and their applications, see Brenti [6] or Stanley [19]. In particular, unlike the case of $S P M$, where a configuration is represented by a partition, we show that each configuration in GSPM corresponds to a unimodal sequence. Amongst other results, using general structure theory of discrete dynamical system, we establish an order structure over the set of all unimodal sequences of the same weight.

The paper is structured as follows. We first recall some basic definitions of lattice theory and the theory of unimodal sequences. Basic results about the two models under study $S P M$ and $L_{B}$ are reviewed in Section 2. We then concentrate on the two models $G S P M$ and $G L_{B}$ and study their structures in Section 3. Finally, we consider a restricted model of $G L_{B}$ which defines a lattice structure over the set of all unimodal sequences of a given weight.

\section{Preliminaries}

In this section, we recall basic definitions and results used in the rest of the paper. 


\subsection{LATTICE THEORY}

An order relation is a binary relation $\leq$ over a set, such that for all $x, y$ and $z$ in this set, $x \leq x$ (reflexivity), $x \leq y$ and $y \leq z$ implies $x \leq z$ (transitivity), and $x \leq y$ and $y \leq x$ implies $x=y$ (antisymmetry). Such a relation is often called a partial order (or order). The set is then a partially ordered set or, for short, a poset.

A lattice is a poset such that any two elements $a$ and $b$ have a least upper bound (called supremum of $a$ and $b$ and denoted by $\sup (a, b)$ ), and a greatest lower bound (called infimum of $a$ and $b$ and denoted by in $f(a, b)$ ). Lattices are strongly structured sets, and many results are known about them. For example, efficient coding and algorithms are known for lattices. For more details, see, e.g., [8,17].

\subsection{Partitions And (Generalized) Unimodal SEQUenCE}

We recall that a partition of an integer $n$ is a sequence of non-increasing positive integers $a=\left(a_{0}, \ldots, a_{m}\right)$ such that $a_{0}+\ldots+a_{m}=n$. The set $\mathcal{P}(n)$ of all partitions of $n$ is equipped with a partial order, called dominance ordering, defined as follows. Given two partitions $a$ and $b$ of $n$, we have $a \geq b$ if the suffix sums of $a$ are smaller than that of $b$, i.e. $\quad \sum_{i>j} a_{i} \leq \sum_{i>j} b_{i}$ for all $j \geq 0$ [7]. Note that this definition still make sense even if $a$ and $b$ are partitions of different integers $m$ and $n$ respectively, where $m \geq n[16]$.

By a unimodal sequence of $n$, we means a positive integers sequence $a=$ $\left(a_{0}, a_{1}, \ldots, a_{m}\right)$ such that $a_{0}+\ldots+a_{m}=n$, and for some $j, a_{0} \leq a_{1} \ldots \leq$ $a_{j} \geq a_{j+1} \ldots \geq a_{m}$. We call $n$ the weight of $a$.

Now define a generalized unimodal sequence of $n$ to be a sequence $a=\left(a_{k}, a_{k+1}\right.$, $\left.\ldots, a_{l}\right)$ such that the sequence $b=\left(b_{0}, \ldots, b_{l-k}\right)$, with $b_{i}=a_{k+i}$ for all $1 \leq i \leq$ $l-k$, is a unimodal sequence of $n$, (by convention, $a_{i}=0$ for all $i<k$ or $i>l$ ). In other words, $a$ is a generalized unimodal sequence of $n$ if $a$ is obtained from a unimodal sequence $b$ of $n$ by adding all of its indices with the same integer. In this case, we say $b$ is the form of $a$. The first index $k$ (which can be positive or negative) is called the position of $a$. We define the center $c(a)$ of $a$ to be the unique integer $q$ such that $a_{q-1}<a_{q} \geq a_{q+1}$, and the height $h(a)$ of $a$ to be the value $a_{q}$. It is clear that each generalized unimodal sequence is determined uniquely by its form and its position, or by its form and its center. For an index $i$, the left subsequence $a_{<i}$ (resp. right subsequence $a_{\geq i}$ ) of $a$ at position $i$ is the sequence $\left(a_{i-1}, a_{i-2}, a_{i-3}, \ldots, a_{k}\right)$ (resp. $\left.\left(a_{i}, a_{i+1}, a_{i+2}, \ldots, a_{l}\right)\right)$. And one can write $a=\left(a_{<i}, a_{\geq i}\right)$. If these two sequences are decreasing, we say that $a$ can be decomposed (to two partitions) at position $i$.

At the end, we call initial configuration the partition (unimodal sequence) $(n)$ (at position 0 ).

\subsection{SAND PILES MODEL AND BRYLAWSKI'S MODEL}

In the theory of discrete dynamical model, one model is defined by its configurations and by it(s) transition rule(s). A chain is a sequence of transitions. 
A configuration $b$ is called reachable from another configuration $a$ if $b$ is obtained from $a$ by a chain. Usually, one starts with a distinguished configuration, also called the initial configuration, and the set of all reachable configurations from it is called the configuration space of the model under study. A fixed point of this model is a reachable configuration on which no transition can be applied. In this paper, all models have $(n)$ as the initial configuration.

Let us give here the formal definitions of two famous models Sand Piles Model $(S P M)$ and Brylawski model $\left(L_{B}\right)$ and their basic properties. Their rules are defined as follows.

Definition 1. SPM is a discrete dynamical model with the following vertical rule: $\left(, \ldots, a_{i}, a_{i+1}, \ldots\right) \rightarrow\left(, \ldots, a_{i}-1, a_{i+1}+1, \ldots\right)$ if $a_{i} \geq a_{i+1}+2$.

Definition 2. $L_{B}$ is a discrete dynamical model with vertical rule and the following horizontal rule: $(, \ldots, p+1, p, p, \ldots, p, p-1, \ldots) \rightarrow(, \ldots, p, p, \ldots, p, p, \ldots)$.

Brylawski proved that the configuration space $L_{B}(n)$, which contains all reachable configurations from $(n)$ by vertical and horizontal rules, is nothing but the set of all partitions of $n$.

In the case of the configuration space $\operatorname{SPM}(n)$, we have the following $S P M$ condition:

Lemma 1 ([13]). A partition is reachable from the initial partition ( $n)$ by SPM transitions if and only if it do not contain any subsequence of the form $(p, p, p)$ or $(p, p, p-1, p-2, \ldots, q+1, q, q)$ (with $0<q<p)$.

In these two models, we have that a configuration $b$ is reachable from another one $a$ if and only if $b$ is smaller than or equal to $a$ by dominance ordering and these models are proved to have a lattice structure $[7,13]$. Therefore each model has its own unique fixed point. The unique fixed point of $L_{B}(n)$ is nothing but the partition $(1,1, \ldots, 1)$. And below there is the $S P M$ fixed point condition.

Lemma 2 ([13]). For an integer $n$, let $0 \leq k^{\prime} \leq k$ be two unique integers such that $n=\frac{k(k+1)}{2}+k^{\prime}$. Then the fixed point of $\operatorname{SPM}(n)$ is the partition $(k, k-$ $\left.1, \ldots, k^{\prime}+1, k^{\prime}, k^{\prime}, k^{\prime}-1, \ldots, 1\right)$.

\section{General sand piles model and general BRYLAWSKI'S MODEL}

\subsection{Definitions of two models $G S P M$ And $G L_{B}$}

It is natural to consider transition rules in two sides, so we defined general models of $S P M$ and $L_{B}$ as follows.

Definition 3. The model GSPM has the following (right and left vertical rules respectively (see Fig. 1):

$$
\begin{aligned}
& \left(, \ldots, a_{i}, a_{i+1}, \ldots\right) \rightarrow\left(, \ldots, a_{i}-1, a_{i+1}+1, \ldots\right) \text { if } a_{i} \geq a_{i+1}+2 \text { or } \\
& \left(, \ldots, a_{i-1}, a_{i}, \ldots\right) \rightarrow\left(, \ldots, a_{i-1}+1, a_{i}-1, \ldots\right) \text { if } a_{i} \geq a_{i-1}+2 .
\end{aligned}
$$




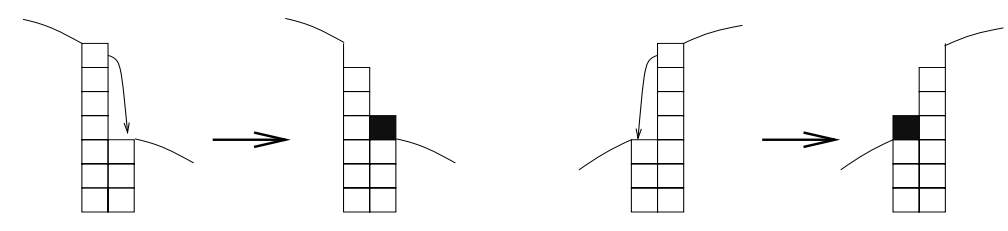

FIgURE 1. Right vertical transition and left vertical transition.
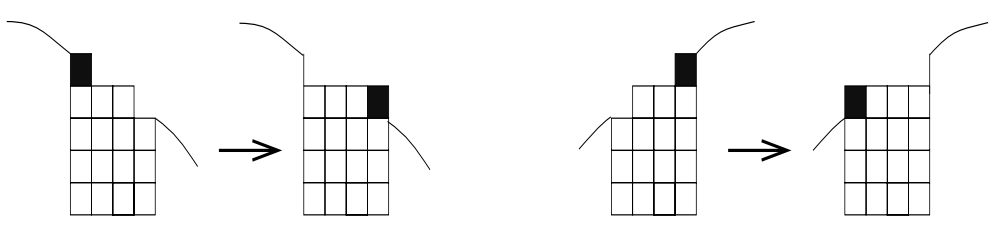

FiguRE 2. Right horizontal transition and left horizontal transition.

Definition 4. The model $G L_{B}$ has vertical rules and the following right and left horizontal rules respectively (see Fig. 2):

$$
\begin{aligned}
& (, \ldots, p+1, p, p, \ldots, p, p-1, \ldots) \rightarrow(, \ldots, p, p, \ldots, p, p, \ldots) \text { or } \\
& (, \ldots, p-1, p, p, \ldots, p, p+1, \ldots) \rightarrow(, \ldots, p, p, \ldots, p, p, \ldots) .
\end{aligned}
$$

We write $a \stackrel{i}{\longrightarrow} b$ for a transition at position $i$. And for each integer $n$, we write $G S P M(n)$ (resp., $\left.G L_{B}(n)\right)$ for the configuration space of $G S P M$ (resp. $G L_{B}$ ) with the initial configuration $(n)$.

Moreover, we define horizontal energy of each configuration $a$ the quantity

$$
E_{H}(a)=\sum_{p} p\left|\left\{a_{i}>p\right\}\right|
$$

It is clear that a horizontal transition decreases this energy by exactly one, and a vertical transition decreases this energy by at least one. This implies that $G S P M(n)$ and $G L_{B}(n)$ have no cycle and then they have an order structure as follows. In $G S P M(n)$ (resp. $G L_{B}(n)$ ), a configuration $b$ is smaller than a configuration $a$, and we write $b \leq_{G S P M} a$ (resp. $\left.b \leq_{G L_{B}} a\right)$, if $b$ is reachable from $a$ by iterating $G S P M$ transitions (resp. $G L_{B}$ transitions). An example of the poset $G S P M$ for $n=6$ is given in Figure 3 .

\subsection{Characterization of Reachable Configurations}

Our first result explains that all configurations of the two models considered can be represented by generalized unimodal sequences. 


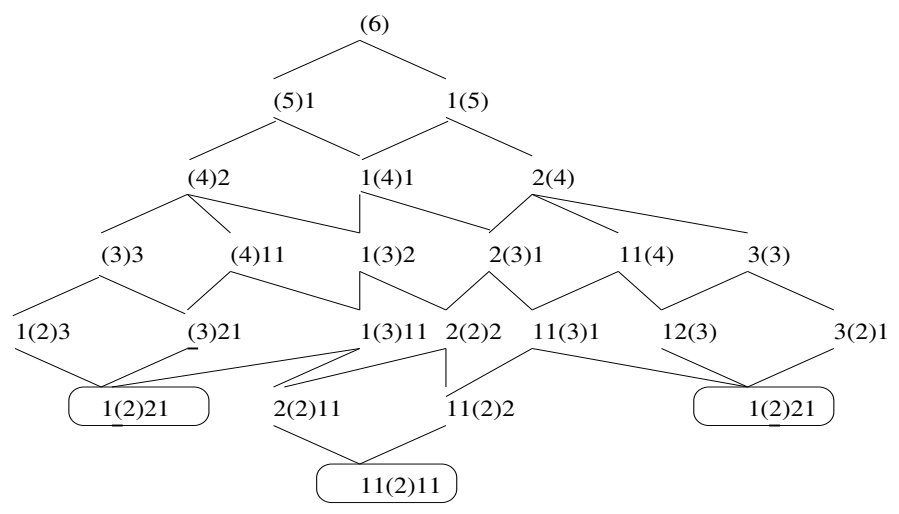

Figure 3. The poset $G S P M(6)$, for each configuration, the integer at position 0 is parenthesed.

Lemma 3. Any configuration of $G S P M(n)$ (or $\left.G L_{B}(n)\right)$ is a generalized unimodal sequence.

Proof. By noting that the initial configuration of these two models is the generalized unimodal sequence $(n)$, it is sufficient to show that for any transition $a \stackrel{i}{\longrightarrow} b$, if $a$ is a generalized unimodal sequence, then $b$ is also a generalized unimodal sequence. Let $q$ be the center of $a$. Let us consider the case where the transition $a \stackrel{i}{\longrightarrow} b$ is a right one, the other case is proved similarly. If $i \neq q$ then $b$ is a generalized unimodal sequence of center $q$ and of same height as $a$. If $i=q, b_{q}$ is still greater than or equal to $b_{q+1}$, and because $q$ is the center of $a$ then $a_{q}>a_{q-1}$, hence $b_{q} \geq b_{q-1}$. Thus $b$ is still a generalized unimodal sequence, and of height $h(a)-1$.

Conversely, note that only certain generalized unimodal sequences are configurations of $\operatorname{GSPM}(n)$, and our purpose is to describe the criterion of such configurations.

Recall that a generalized unimodal sequence is determined by its form and its position. We give first the criterion for the form of reachable configurations of $G S P M$, called GSPM condition, by generalizing the SPM-condition.

Lemma 4. A unimodal sequence $a$ of $n$ is the form of a configuration of $G S P M(n)$ if and only if a has a decomposition $a=\left(a_{<i}, a_{\geq i}\right)$ where $a_{<i}$ and $a_{\geq i}$ are two partitions satisfying SPM condition.

Proof. Let $a$ be a unimodal sequence satisfying $G S P M$ condition. We prove that there exists a generalized unimodal sequence $b$ of form $a$ such that $b \in \operatorname{GSPM}(n)$. Let $n-k$ be the weight of $a_{<i}$ and $k$ be the weight of $a_{\geq i}$. Because $a_{<i}$ satisfies $S P M$ condition, then this partition is reachable from the partition $(n-k)$ by $S P M$ rule. Similarly, $a_{\geq i}$ is reachable from the partition (k). If $n-k \leq k$, we 
choose $b$ to be the generalized unimodal sequence of the form $a$ and of position $i$, then $b$ is reachable from the generalized unimodal sequence of the form $(n-k, k)$ at position -1 , which is reachable from the intimal $(n)$ (at position 0 ). Otherwise, we chose $b$ of position $i-1$, and $b$ is reachable from $(n)$ also.

For the converse, we prove that GSPM condition is invariant under GSPM transitions. Let $a \stackrel{j}{\longrightarrow} b$ be such a transition where $a$ satisfies GSPM condition, we need to prove that $b$ also satisfies GSPM condition. Without loss of generality, we can assume that this transition is a right one. There are two possible cases: $j \geq i$ or $j<i$. If $j \geq i$, it is easy to see that $b$ is decomposed to $\left(b_{<i}, b_{\geq i}\right)$ where $b_{<i}=a_{<i}$ and $b_{\geq i}=a_{\geq i} \stackrel{j}{\longrightarrow}$. Then $b_{<i}$ and $b_{\geq i}$ satisfy $S P M$ condition, and then $b$ satisfies GSPM condition. Consider now the case when $j<i$. We have $a_{j+1} \leq a_{j}-2$, so $a_{j+1}$ is not in the partition $a_{<i}$, which implies that $j=i-1$ and $j+1=i$. We take the decomposition $\left(b_{<i-1}, b_{\geq i-1}\right)$ where $b_{<i-1}=a_{<i-1}$ and $b_{>i-1}=\left(a_{i-1}-1, a_{i}+1, a_{i+1}, a_{i+2}, \ldots\right)$. One can verify that $b_{<i-1}$ and $b_{>i-1}$ satisfy $S P M$ condition, then $b$ satisfies $G S P M$ condition.

For a unimodal sequence (resp. generalized unimodal sequence) $a$, a decomposition at position $i$ of $a$ that satisfies GSPM condition is called a GSPM decomposition. Note that $a$ may have some different $G S P M$ decompositions. For example, if $a=(1,3,4,5,5,2,1), a$ has three $G S P M$ decompositions, at position 4, 5, 6 . But we can also claim that $\max \left(a_{i-1}, a_{i}\right)$ is always the height of $a$.

Having a criterion on the possible forms of a reachable configurations, we now turn to their possible positions.

Let $a$ be a generalized unimodal sequence satisfying $G S P M$ condition, and let $a=\left(a_{<i}, a_{\geq i}\right)$ be a $G S P M$ decomposition. We want to give a limit for the index $i$. For this, we introduce the following function $w(i, a)$ :

Definition 5. Let $a=\left(a_{<i}, a_{\geq i}\right)$ be a GSPM decomposition of a generalized unimodal sequence $a$. If $i \geq 0$, we define $f(i, a)$ the partition $s=\left(s_{0}, s_{1}, \ldots\right)$ of minimal weight such that $s$ satisfies SPM condition and that $s_{\geq i}=a_{\geq i}$. Similarly, if $i<0$, we define $f(i, a)$ the generalized unimodal sequence $\left(\ldots, s_{-2}, s_{-1}\right)$ of minimal weight such that $s_{<0}$ satisfies $S P M$ condition and that $s_{<i}=a_{<i}$. The weight of $f(i, a)$ is denoted by $w(i, a)$.

We state the following condition.

Lemma 5. Let a be a generalized unimodal sequence, a belongs to $\operatorname{GSPM}(n)$ if and only if a has a GSPM decomposition at a position $i$ such that $w(i, a) \leq n$.

Proof. We prove for the case $i \geq 0$, the second case will be derived immediately. To prove the necessary condition for elements of $\operatorname{GSPM}(n)$, it is sufficient to show that if $a$ has a $G S P M$ decomposition at position $i$ such that $w(i, a) \leq n$ and if $a \stackrel{j}{\longrightarrow} b$ is a $G S P M$ transition, then $b$ has a similar decomposition too. By using arguments in the proof of Lemma 3, it is easy to see that we can decompose $b$ at position $i$ or $i-1$, in any case $w(i, b)$ or $w(i-1, b)$ are still smaller than or equal to $w(i, a)$. 
Conversely, let us consider a generalized unimodal sequence $a$ satisfying the Lemma condition. Let $t=\left(t_{0}, t_{1}, \ldots\right)$ be a sequence defined by: for all $j \geq 1$, $t_{j}=s_{j}$, and $t_{0}=s_{0}+n-w(i, a)$. By hypothesis, $t_{0}$ is greater than $s_{0}$, and $t$ is a partition of $n$ satisfying $S P M$ condition (see Lemma 1), hence $t$ is reachable from $(n)$. It is easy to check that one can move grain of $t$ at position $0,1, \ldots, i-1$ from right to left, to obtain configuration $a$. So $a$ is reachable from $t$ by GSPM transition, and then reachable from $(n)$.

In order to give a complete characterization of configurations of $G S P M(n)$, let us calculate exactly the value $w(i, a)$ here. For that, we need to define two new notions. A step is a sequence of the form $(p, p-1, \ldots, q+1, q)$ and a slide step is a sequence of the form $(p, p-1, \ldots, q+1, q, q)$, with $p \geq q>0$. By regarding $S P M$ condition, for a generalized unimodal sequence $a$ with a GSPM decomposition at a position $i \geq 0$, one can chose $f(i, a)$ as:

- if $a_{\geq i}$ begins with a slide step then $\left(s_{0}, \ldots, s_{i}\right)$ is a step, that means $f(i, a)=\left(a_{i}+i, \ldots, a_{i}+1, a_{\geq i}\right)$. And $w(i, a)=i a_{i}+\frac{i(i+1)}{2}+\sum_{j \geq i} a_{j} ;$

- otherwise, $\left(s_{0}, \ldots, s_{i}\right)$ is a slide step, that means $f(i, a)=\left(a_{i}+i-\right.$ $\left.1, \ldots, a_{i}+1, a_{i}, a_{\geq i}\right)$. And $w(i, a)=i a_{i}+\frac{i(i-1)}{2}+\sum_{j \geq i} a_{j}$.

Similarly, in the case $a$ has a GSPM decomposition at a position $i<0$, one can calculate $w(i, a)=-i a_{i-1}+\frac{i(i-1)}{2}+\sum_{j<i} a_{j}$ if $a_{<i}$ begins with a slide step, and $w(i, a)=-i a_{i-1}+\frac{i(i+1)}{2}+\sum_{j<i} a_{j}$ otherwise.

We give finally the following GSPM characterization.

Theorem 1. A generalized unimodal sequence a belongs to $G S P M(n)$ if and only if a has a GSPM decomposition at some position $i$ such that:

- if $i \geq 0$ then: $i a_{i}+\frac{i(i+1)}{2}+\sum_{j \geq i} a_{j} \leq n$ if $a_{\geq i}$ begins with a slide step, or $i a_{i}+\frac{i(i-1)}{2}+\sum_{j \geq i} a_{j} \leq n$ otherwise;

- if $i<0$ then: $-i a_{i-1}+\frac{i(i-1)}{2}+\sum_{j<i} a_{j} \leq n$ if $a_{<i}$ begins with a slide step, or $-i a_{i-1}+\frac{i(i+1)}{2}+\sum_{j<i} a_{j} \leq n$ otherwise.

\subsection{Fixed points of $G S P M(n)$}

Regarding the configuration space $G S P M(6)$ in Figure 3, one can see that the poset $\operatorname{GSPM}(n)$ is not a lattice and it has several fixed points. In this section, we will describe all its fixed points.

Let $P$ be a fixed point of $\operatorname{GSPM}(n)$. By Proposition 1, $P$ can be decomposed as $\left(P_{<i}, P_{\geq i}\right)$ where $P_{<i}$ and $P_{\geq i}$ satisfy $S P M$ condition. If $P_{\geq i}$ (resp. $\left.P_{<i}\right)$ is not a $S P M$ fixed point then one can apply on it a right (resp. left) transition. Moreover, if $\left|P_{i}-P_{i-1}\right| \geq 2$ then one grain can move from the higher column to the lower column (between two columns $i$ and $i-1$ ). Hence, if $P_{<i}$ and $P_{\geq i}$ are $S P M$ fixed points and $\left|P_{i}-P_{i-1}\right| \leq 1$, then there exist a fixed point of the same form as $P$. For a complete characterizations about the form and the position of fixed points we state following result. 
Theorem 2. A generalized unimodal sequence $P$ is a fixed point of $G S P M(n)$ if $P$ has a GSPM decomposition at some position $i$ such that:

- $P_{<i}$ and $P_{\geq i}$ are $S P M$ fixed points and $\left|P_{i}-P_{i-1}\right| \leq 1$;

- the height $k$ of $P$ is either $\lfloor\sqrt{n}\rfloor$ or $\lfloor\sqrt{n}\rfloor-1$; and

- the position $i$ satisfies $k+|i| \leq\lfloor\sqrt{2 n}\rfloor$.

Proof. Let $k$ be the height of $P$. The GSPM fixed point of height $k$ of smallest sum is $P_{1}=(1,2, \ldots, k-1, k, k-1, \ldots 2,1)$, and the $G S P M$ fixed point of greatest sum is $P_{2}=(1,2, \ldots, k-1, k, k, k, k, k-1, \ldots, 2,1)$ because of Lemma 1 and 4 . Their sum are respectively $k^{2}$ and $k(k+3)$. So the value of $k$ may be $\lfloor\sqrt{n}\rfloor$ or $\lfloor\sqrt{n}\rfloor-1$.

Let us consider $w(i, P)$ in the case $i \geq 0$, the other case is similar. We have $w\left(i, P_{1}\right)=\frac{(k+i-1)(k+i)}{2}+k$, and $w\left(i, P_{2}\right)=\frac{(k+i)(k+i+1)}{2}+k$. And for all other $G S P M$ fixed point $P, w\left(i, P_{1}\right) \leq w(i, P) \leq w\left(i, P_{2}\right)$. On the other hand, we know that $w(i, P) \leq n$, then we prove that $k+i \leq\lfloor\sqrt{2 n}\rfloor$. In fact, if $k+i>\lfloor\sqrt{2 n}\rfloor$, then $k+i \geq\lfloor\sqrt{2 n}\rfloor+1>\sqrt{2 n}$. Moreover, because $k \geq i$ then $k>\frac{\sqrt{2 n}}{2}$. Hence $w(i, P) \geq w\left(i, P_{1}\right)=\frac{(k+i-1)(k+i)}{2}+k>\frac{(\operatorname{sqrt} 2 n-1) \sqrt{2 n}}{2}+\frac{s q r t 2 n}{2}=n$, which is a contradiction. Furthermore, from the above statement, we see that the maximal value for $k+i$ is $\lfloor\sqrt{2 n}\rfloor$.

\subsection{Longest AND SHORTEST CHAINS IN $G S P M(n)$}

To calculate the length of a sequence of vertical rules, we introduce the vertical energy of a generalized unimodal sequence $a$, denoted by $E_{V}(a)$, as follows:

$$
E_{V}(a)=\sum_{\forall i} a_{i}|i|
$$

Clearly, the vertical energy of the initial configuration $(n)$ is equal to 0. Let us consider the energy difference $E_{V}(b)-E_{V}(a)$ for a vertical transition $a \stackrel{i}{\longrightarrow} b$. If this transition is a right transition and $i \geq 0$ or if this transition is a left one and $i \leq 0$, we have $E_{V}(b)-E_{V}(a)=1$. In other case (a right transition at a negative position $i$ or a left transition at a positive position $i$ ), we have $E_{V}(b)-E_{V}(a)=-1$

Therefore, a shortest chain in $\operatorname{GSPM}(n)$ is a chain from $(n)$ to a fixed point of smallest vertical energy which contains only transitions of the first type. For that, we can choose a fixed point of center 0 .

On the other hand, a longest chain contains a maximum of transitions of the second type. One of these chains can be chosen from $(n)$ to a fixed point $P=$ $\left(P_{<i}, P_{\geq i}\right)$ of maximal distance $i=\lfloor\sqrt{2 n}\rfloor-\lfloor\sqrt{n}\rfloor$, via the partition $f(i, P)$ (see Lem. 5).

\subsection{The Structure of The $G L_{B}$ MODEL}

One can now ask about configurations of the general model $G L_{B}(n)$. As for the classical model $L_{B}$ (all partitions of $n$ are reachable in $L_{B}(n)$ model), and the 
above properties of $G S P M$, we establish similar properties for $G L_{B}$. The result on the form of reachable configuration $G L_{B}(n)$ is straightforward:

Lemma 6. Every unimodal sequence can be the form of a configuration of $G L_{B}(n)$.

We give now the characterization for position of elements of $G L_{B}(n)$.

Proposition 1. A generalized unimodal sequence a belongs to $G L_{B}(n)$ if and only if:

or

$$
c(a) \cdot h(a) \leq \sum_{i<c(a)} a_{i} \quad \text { if } c(a) \geq 0
$$

$$
(-c(a)) \cdot h(a) \leq \sum_{i>c(a)} a_{i} \quad \text { if } c(a)<0 .
$$

Proof. Suppose that $c(a) \geq 0$. Similarly to Lemma 5 , let us consider the partition $L(a)=\left(l_{0}, l_{1}, \ldots\right)$ such $l_{0}=l_{1}=\ldots l_{c}=h$ and for all $j \geq c, l_{j}=a_{j}$, where $h=h(a)$ and $c=c(a)$. Let $w(a)$ be the weight of $L(a)$. One can state that $a$ belongs to $G L_{B}(n)$ if and only if $n \geq w(a)$. Which implies the result.

The proof is similar for the case $c(a)<0$.

As for the fixed points of $G L_{B}(n)$, the situation is simpler. It is evident that the form of all fixed points are $(1, \ldots 1)$, and then their center are always their first position. We have:

Corollary 1. $G L_{B}(n)$ has $n$ fixed points of form $(1, \ldots, 1)$ where the first position can take value from $-n+1$ to 0 .

\subsection{LONGEST AND SHORTEST CHAINS IN $G L_{B}(n)$}

Observe that one needs one (vertical) transition to move one grain from position 0 to position 1 or -1 . Otherwise, if $a_{0}>2$ and for $|i| \leq 2$, one needs at least a double transition to move one grain from position 0 to position $i$ : a vertical transition from position 0 to position 1 (resp. -1) and a horizontal transition from position 1 (resp. -1 ) to position $i$ if $i \geq 2$ (resp. if $i \leq-2$ ). Therefore, a shortest chain is composed of $n-4$ double transitions to move one grain from position 0 to position $i \neq 1,-1$, one vertical transition to move one grain to position 1 , another to position -1 , a horizontal transition from position 0 to move one grain of height 2 to a position $i$ and. So its length is equal to $3+2(n-4)=2 n-5$.

For longest chain, we prove that the result in $G L_{B}$ is the same at in $L_{B}$. Let us first consider a surjection $\varphi$ from $G L_{B}(n)$ to $L_{B}(n)$ : for each unimodal sequence $a, \varphi(a)$ is the partition obtained from $a$ by resorting parts of $a$ in decreasing order. It is clear that $\varphi$ is subjective. By the way, for any transition $a \rightarrow b$ in $G L_{B}, \varphi(b)$ is smaller than $\varphi(a)$ by dominance ordering, so $\varphi(b)$ can be obtained from $\varphi(a)$ by a chain in $L_{B}$ [7]. It implies that for every chain in $G L_{B}(n)$, one can find a longer chain in $L_{B}(n)$. Therefor the maximal length in $G L_{B}(n)$ is smaller than that in $L_{B}(n)$. However, one can consider an injection $\psi$ from $L_{B}(n)$ to $G L_{B}(n)$ 
such that $\psi(a)=a$, so a chain in $L_{B}(n)$ is also a chain in $G L_{B}(n)$. Hence, longest chains in $G L_{B}(n)$ is longest chains in $L_{B}(n)$. For more detail on longest chains in $L_{B}(n)$, one can see the result of Greene and Kleitmann [14].

\section{UNIMODAL SEQUENCES MODEL}

In this section, we define a discrete model, induced from $G L_{B}$, which generates each unimodal sequence exactly once. We then give combinatorial properties and lattice structure of this model. Note that, different from the usual definition of unimodal sequence (whose the first position is 0 ), in this section, we call a unimodal sequence a generalized unimodal sequence of center 0 .

\subsection{THE MODEL}

As one can see from the above section, in $G L_{B}(n)$, there are many configurations (generalized unimodal sequences) of the same form; in other words, a unimodal sequence may "occur" at different positions. The purpose of this section is to construct a model where every configurations are of center 0 , so that its they "occur" at most once. This model is an induced model of $G L_{B}$ model by adding the following condition: $a_{0} \geq a_{1}$ and $a_{0}>a_{-1}$. For further detail, all transitions rest the same transitions as in $G L_{B}$ except at position 0 . At this position, we have the following possible transitions:

$$
\begin{aligned}
& a \stackrel{0}{\longrightarrow}\left(\ldots, a_{0}-1, a_{1}+1, \ldots\right) \text { if } a_{0} \geq a_{1}+2, a_{0}>a_{-1}+1 \\
& a \stackrel{0}{\longrightarrow}\left(\ldots, a_{0}-1, a_{1}, \ldots, a_{k-1}, a_{k}+1, \ldots\right) \\
& \quad \text { if } a_{0}=a_{1}+1=\ldots=a_{k-1}+1=a_{k}+2, a_{0}>a_{-1}+1 \\
& a \stackrel{0}{\longrightarrow}\left(\ldots, a_{-1}+1, a_{0}-1, \ldots\right) \text { if } a_{0} \geq a_{1}+1, a_{0}>a_{-1}+2 \\
& a \stackrel{0}{\longrightarrow}\left(\ldots, a_{-k}+1, a_{-k+1}, \ldots, a_{-1}, a_{0}-1, \ldots\right) \\
& \quad \text { if } a_{0}-1=a_{-1}+1=\ldots=a_{-k+1}+1=a_{-k}+2, a_{0}>a_{1} .
\end{aligned}
$$

Let us call this induced model the Unimodal Sequences Model and denote it by $U S M$. The configuration space, i.e. the set of all reachable configurations from the initial configuration $(n)$, is denoted by $U S M(n)$. Just as in above section, $U S M(n)$ is a poset with partial order $b \leq_{U S M} a$ if $b$ is reachable from $a$. We are now going to represent this order combinatorically, that means an order over unimodal sequences. To do that, let us first give some notations: for a unimodal sequence $a$ and for all positive integer $i$, the suffix sum of $a$ at position $i$ is $A_{i}=$ $\sum_{j \geq i} a_{j}$ and at position $-i$ is $A_{-i}=\sum_{j \leq-i} a_{j}$. We define dominance ordering $\leq$ dom as follows:

$$
a \geq_{d_{o m}} b \text { if and only if for all integer } i \neq 0, A_{i} \leq B_{i},
$$

(it is easy too see that this is an order relation with the maximum $(n)$ ). 


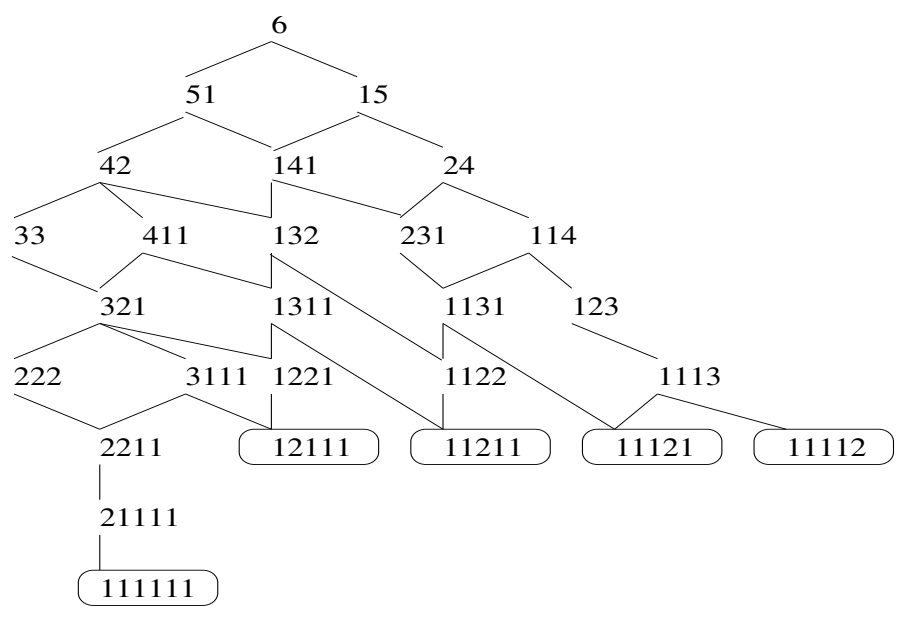

Figure 4 . The poset $U S M(6)$, its fixed points are bordered by a circle.

Lemma 7. Let $a$ and $b$ be two unimodal sequences, then $b \leq_{d o m} a$ if and only if $b \leq_{U S M} a$.

Proof. It is evident to see that if $b$ is obtained from $a$ by a transition, then $b \leq_{\text {dom }} a$, so $b \leq_{U S M} a$ implies $b \leq_{\text {dom }} a$.

Now, suppose that $b \leq_{\text {dom }} a$, we want to prove that $b \leq_{U S M} a$. It is sufficient to find a unimodal sequence $c$ such that $a \rightarrow c$ by $U S M$ transition and $c \geq_{\text {dom }} b$.

Let us consider the case $a_{\geq 0} \neq b_{\geq 0}$. Note that $a_{0}+A_{1}=n-A_{-1} \geq n-B_{-1}=$ $b_{0}+B_{1}$. Hence the partition $a_{\geq 0}$ is greater than the partition $b_{\geq 0}$ by dominance ordering. So one can apply a transition at one position $j \geq 0$ of $a$ to obtain a new unimodal sequence $c$, and this unimodal sequence is still greater than $b$ by dominance ordering. The only inconvenient case is when one need to apply a transition at position 0 of $a$ but $a_{0}=a_{-1}+1$. In this case, $b_{0}<a_{0}$, then $b_{-1}<b_{0} \leq a_{-1}$. Let $k$ be the greatest positive integer such that $a_{-k}=a_{-1}$. Let $-l$ be the first position that one grain of $a$ can move from the position $-k$ to. We have $A_{-1} \leq B_{-1}$, then $A_{-k}<B_{-k}, A_{-l}<B_{-l}$, it implies that $C_{-l} \leq B_{-l}$ and for all $i, C_{i} \leq B_{i}$. So $c$ satisfies our purpose.

In the case $a_{\geq 0}=b_{\geq 0}$, we have $a_{\leq 0} \neq b_{\leq 0}$, the result is proved by the same way.

Note that the initial unimodal sequence $(n)$ is greatest by dominance ordering, so using this Lemma, we conclude that every unimodal sequence is reachable from this initial one. We have proved the following.

Theorem 3. The configuration space $\operatorname{USM}(n)$ is a poset of all unimodal sequences of center 0 where $b$ is reachable from $a$ if and only if $b \leq_{\operatorname{dom}} a$. 
Due from this Theorem, from now on, we means dominance ordering while saying order between unimodal sequences, or between partitions.

An example of $U S M(n)$ is given in Figure 4. Although this poset has no lattice structure, we will prove that its structure is in fact a sup semilattice (Corollary 5). Recall that a poset is a sup semilattice if every pair of its elements has a supremum.

First of all, let us prove a result on the supremum of two partitions:

Lemma 8. Let $a=\left(a_{0}, a_{1}, \ldots\right)$ and $b=\left(b_{0}, b_{1}, \ldots\right)$ be two partitions of $n$. Let $c=\left(c_{0}, c_{1}, \ldots\right)$ is the supremum of $a$ and $b$. Then $c_{0}=\max \left(a_{0}, b_{0}\right)$.

Proof. Keep in mind the notation of $A_{i}$. In [7,16], the authors gave formula for the infimum of two partitions, but not its supremum. But, using the symmetric structure of Brylawski's lattice, one can define also the supremum. For a given partition $a$ of $n$, the dual (or conjugate) partition $a^{*}=\left(a_{0}^{*}, a_{1}^{*}, \ldots\right)$ is defined as $a_{i}^{*}=\mid\left\{a_{j} \geq i+1\right\}$. The maximum part $a_{0}$ of $a$ is nothing but the number of parts of $a^{*}$. Let $d$ be the infimum of $a^{*}$ and $b^{*}$, then $d=c^{*}$. Hence $c_{0}$ is the number of parts of $d$. Let $k$ be the maximum of number of parts of $a^{*}$ and the number of parts of $b^{*}$. For $j>k, \max \left(A_{j}^{*}, B_{j}^{*}\right)=0$, and then $D_{j}^{*}=0$; and $D_{k}^{*}=\max \left(A_{K}^{*}, B_{k}^{*}\right) \neq 0$, so $d_{k} \neq 0$, and the number of parts of $d^{*}$ is $k$. That means $c_{0}=k$, which is the maximum of $a_{0}$ and $b_{0}$.

To study the strong structure of $\operatorname{USM}(n)$, we will prove that it is an union of lattices. To achieve this, we first give the description of its fixed points.

Corollary 2. In poset $\operatorname{USM}(n)$, there are $n-2$ fixed points of the form

$$
P_{k}=(1, \ldots, 1,2,1, \ldots, 1),
$$

consisting of $k$ entries 1 with $1 \leq k \leq n-2$, followed by a 2 , and $n-2-k$ entries 1 again; and another fixed point $P_{0}=(1, \ldots 1)$.

For each $k=0,1, \ldots, n-2$, let $I_{k}=\left[(n), P_{k}\right]$ be the set of all unimodal sequences which are greater than or equal to $P_{k}$. Note that if $k=0$, the interval $I_{0}$ is nothing but the Brylawski's lattice $L_{B}(n)$, and that for all $k \geq 1$ :

$$
I_{k}=\left\{a \text { such that } A_{1} \leq n-2-k \text { and } A_{-1} \leq k\right\} .
$$

Theorem 4. For each $0 \leq k \leq n-2$, the interval $I_{k}$ is a lattice.

Proof. We just need to prove the statement for $k \geq 1$. Given two unimodal sequences $a$ and $b$ of $I_{k}$, we will construct their infimum $c$. The idea is to define a unimodal sequence $c$ such that $C_{1}=\max \left(A_{1}, B_{1}\right), C_{-1}=\max \left(A_{-1}, B_{-1}\right)$, and $c_{0}=n-C_{1}-C_{-1}$.

First of all, put $c_{0}=n-C_{1}-C_{-1}$. We now establish $c_{\geq 1}$ and $c_{<0}$ by noting that $c_{0}$ must satisfy: $c_{-1}<c_{0} \geq c_{1}$ ).

Let us consider two partitions: $\left(a_{1}, a_{2}, \ldots\right)$ - partition of $A_{1}$, and $\left(b_{1}, b_{2}, \ldots\right)$ - partition of $B_{1}$. Let $\left(d_{1}, d_{2}, \ldots,\right)$ be the supremum of these two partitions by dominance ordering. So, we have: $d_{1}+d_{2}+\ldots=\max \left(A_{1}, B_{1}\right)=C_{1}$, and for all $i>0, D_{i}=\max \left(A_{i}, B_{i}\right)$. 
If $d_{1} \leq c_{0}$, we can take $\left(c_{1}, c_{2}, \ldots\right)=\left(d_{1}, d_{2}, \ldots\right)$.

In the case $d_{1}>c_{0}$, we consider the following set

$S=\left\{s=\left(s_{1}, s_{2}, \ldots\right) \| s\right.$ is a partition of $C_{1}$, such that $s_{1} \leq c_{0}$ and $\left.s \leq d\right\}$.

Because the set of all partitions of an integer ordered by dominance ordering is a lattice, so there exist a supremum for set $S$, and we take $\left(c_{1}, c_{2}, \ldots\right)$ to be this supremum. By using Lemma 8 , we have $c_{1}$ is smaller than or equal to $c_{0}$.

The partition $\left(c_{-1}, c_{-2}, \ldots\right)$ is defined in the same way by noting that $c_{-1}<c_{0}$.

The sequence $c$ defined as above is a clearly an element of $I_{k}$ and $c$ is the infimum of $a$ and $b$ (it is easy to verify that if $c^{\prime}$ is smaller than $a$ and $b$ then $c^{\prime}$ is smaller than $c$ by $\left.\leq_{U S M}\right)$. The interval $I_{k}$, which has a greatest element $(n)$ and which is closed to the operation infimum, is then a lattice. This proves the Theorem.

Remark 1. The infimum of two elements $a$ and $b$ of $I_{k}$ is defined independently of $k$. That means that if $a$ and $b$ belong to $I_{k}$ and to $I_{l}$ both, then $i n f_{I_{k}}(a, b)$ is the same as inf $f_{I_{l}}(a, b)$. So we can define inf $(a, b)$ in $U S M(n)$ the infimum of a and $b$ in a certain $I_{k}$ if $a, b \in I_{k}$.

Corollary 3. For two unimodal sequences $a$ and $b$, its infimum in $U S M(n)$ exists if and only if either $A_{-1}=B_{-1}=0$ or $\left(A_{-1}+B_{1} \leq n\right.$ and $\left.A_{1}+B_{-1} \leq n\right)$.

Proof. We claim that $a$ and $b$ has an infimum if and only if they belong to the same $I_{k}$ for some $k \geq 0$. In fact if $c$ is the infimum of $a$ and $b$, and $c$ belongs to $I_{k}$ then $a$ and $b$ belong to $I_{k}$ too. Conversely, if $a$ and $b$ belong to the same $I_{k}$, then its infimum exists by Theorem 4 .

Finally, the case $I_{0}$ corresponds to the condition that $A_{-1}=0$ and $B_{-1}=0$. Otherwise, $a \in I_{k}$ if and only if $A_{1} \leq n-2-k$ and $A_{-1} \leq k$, i.e. $\quad A_{-1} \leq k \leq$ $n-2-A_{1}$. So $a$ and $b$ belong to the same $I_{k}$ for some $k$ if and only if $A_{-1}+B_{1} \leq n$ and $A_{1}+B_{-1} \leq n$.

Theorem 4 implies also the following result on the structure of the poset $U S M(n)$.

Corollary 4. The poset USM(n) is a sup semilattice.

Proof. Let $a$ and $b$ be two unimodal sequences, we prove by contradiction that $\sup (a, b)$ exists. Assume that there exists (at least) two minimal elements of $U S M(n)$ which are greater than $a$ and $b$, say $c$ and $d$. Let $P_{k}$ be a fixed point smaller than $a$, we have $c, d \in I_{k}$, it implies that there exists $e=\inf (c, d)$. The unimodal sequence $e$ is greater than $a$ and $b$, and smaller than $c$ and $d$, which is a contradiction.

To give a lattice structure over the set of all unimodal sequences, we introduce the poset $\overline{U S M(n)}$ obtained from $U S M(n)$ by adding order relations as follows: for all $1 \leq k \leq n-2$, we define that $P_{k}$ is greater than $P_{0}$.

Corollary 5. $\overline{U S M(n)}$ has a lattice structure. 
Proof. The supremum of two elements is already defined. The infimum of two unimodal sequences $a$ and $b$ is defined as follows: if there exists $k$ such that $a, b \in I_{k}$, then $\inf (a, b)$ is defined as in Theorem 4, else $\inf (a, b)=P_{0}$.

And bellows is the relationship between the initial Brylawski's lattice and our lattice $\overline{\operatorname{USM}(n)}$

Corollary 6. For all integer $n, L_{B}(n)$ is sub-lattice of $\overline{\operatorname{USM}(n)}$.

Proof. Brylawski's lattice $L_{B}(n)$ is nothing but the interval $I_{0}$.

At the end, by applying the same argument as in Section 2, one can see that the longest chains in $\operatorname{USM}(n)$ are longest chains in $L_{B}(n)$.

\section{Conclusion}

In this paper, we have defined a natural generalization of two classical discrete models $S P M$ and $L_{B}$ and studied their structure. It seems that among many generalizations of $S P M$ model, our generalization is one of the most natural and this gives a strong structure order. We then introduced the dominance ordering and its lattice structure over the set of all unimodal sequences, an important combinatorial object. We believe that this order is an interesting and meaningful extension of the lattice of partitions, and we hope that some work will be done to extent the classical results to this case.

\section{Note added}

After this paper was ready for publication we have learned of reference [10], where the authors give independent proofs for similar results of Lemma 4 and Theorem 2, using a different method.

\section{REFERENCES}

[1] R. Anderson, L. Lovász, P. Shor, J. Spencer, E. Tardos, and S. Winograd. Disks, ball, and walls: analysis of a combinatorial game. Amer. Math. Monthly 96 (1989) 481-493.

[2] P. Bak, C. Tang, and K. Wiesenfeld. Self-organized criticality. Phys. Rev. A 38 (1988) 364-374.

[3] J. Bitar and E. Goles. Paralel chip firing games on graphs. Theoret. Comput. Sci. 92 (1992) 291-300.

[4] A. Bjorner, L. Lovász, and W. Shor. Chip-firing games on graphes. Eur .J. Combin. 12 (1991) 283-291.

[5] A. Bjorner and G. Ziegler. Introduction to greedoids. Matroid applications, N. White, Ed. Cambridge University Press (1991) 284-357.

[6] F. Brenti. Log-concave and unimodal sequences in algebra, combinatorics and geometry: an update. Contemporary Mathematics 178 (1994) 71-84.

[7] T. Brylawski. The lattice of interger partitions. Discrete Mathematics 6 (1973) 201-219.

[8] B.A. Davey and H.A. Priestley. Introduction to Lattices and Order. Cambridge University Press (1990).

[9] E. Duchi, R. Mantaci, D. Rossin, and H.D. Phan. Bidimensional sand pile and ice pile models. PUMA 17 (2006) 71-96. 
[10] E. Formenti, B. Masson, and T. Pisokas. Advances in symmetric sandpiles. Fundamenta Informaticae 20 (2006) 1-22.

[11] E. Goles and M.A. Kiwi. Games on line graphes and sand piles. Theoret. Comput. Sci. 115 (1993) 321-349.

[12] E. Goles, M. Morvan, and H.D. Phan. Lattice structure and convergence of a game of cards. Ann. Combin. 6 (2002) 327-335.

[13] E. Goles, M. Morvan, and H.D. Phan. Sandpiles and order structure of integer partitions. Discrete Appl. Math. 117 (2002) 51-64.

[14] C. Greene and D.J. Kleitman. Longest chains in the lattice of integer partitions ordered by majorization. Eur. J. Combin. 7 (1986) 1-10.

[15] M. Latapy, R. Mantaci, M. Morvan, and H.D. Phan. Structure of some sand piles model. Theoret. Comput. Sci, 262 (2001) 525-556.

[16] M. Latapy and H.D. Phan. The lattice of integer partitions and its infinite extension. To appear in Discrete Mathematics (2008).

[17] Ha Duong Phan. PhD thesis. Université Paris VII (1999).

[18] J. Spencer. Balancing vectors in the max norm. Combinatorica 6 (1986) 55-65.

[19] R. Stanley. Log-cocave and unimodal sequences in algebra, combinatorics and geometry. Graph theory and its applications: East and West (Jinan 1986). Ann. New York Acad. Sci. 576 (1989). 